\title{
Cerebellar Watershed Injury in Children
}

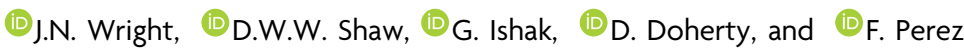

\begin{abstract}
BACKGROUND AND PURPOSE: Focal signal abnormalities at the depth of the cerebellar fissures in children have recently been reported to represent a novel pattern of bottom-of-fissure dysplasia. We describe a series of patients with a similar distribution and appearance of cerebellar signal abnormality attributable to watershed injury.
\end{abstract}

MATERIALS AND METHODS: Twenty-three children with MR imaging findings of focal T2 prolongation in the cerebellar gray matter and immediate subjacent white matter at the depth of the fissures were included. MR imaging examinations were qualitatively analyzed for the characteristics and distribution of signal abnormality within posterior fossa structures, the presence and distribution of volume loss, the presence of abnormal contrast enhancement, and the presence and pattern of supratentorial injury.

RESULTS: 12 prolongation was observed at the depths of the cerebellar fissures bilaterally in all 23 patients, centered at the expected location of the deep cerebellar vascular borderzone. Diffusion restriction was associated with MR imaging performed during acute injury in 13/ 16 patients. Five of 23 patients had prior imaging, all demonstrating a normal cerebellum. The etiology of injury was hypoxic-ischemic injury in $17 / 23$ patients, posterior reversible encephalopathy syndrome in $3 / 23$ patients, and indeterminate in $3 / 23$ patients. Twenty of 23 patients demonstrated an associated classic parasagittal watershed pattern of supratentorial cortical injury. Injury in the chronic phase was associated with relatively preserved gray matter volume in 8/15 patients, closely matching the published appearance of bottom-of-fissure dysplasia.

CONCLUSIONS: In a series of patients with findings similar in appearance to the recently described bottom-of-fissure dysplasia, we have demonstrated a stereotyped pattern of injury attributable to cerebellar watershed injury.

ABBREVIATIONS: $\mathrm{HII}=$ hypoxic-ischemic injury; PRES = posterior reversible encephalopathy syndrome; SCA = superior cerebellar artery

$\mathbf{F}^{\circ}$ ocal signal abnormalities at the depth of the cerebellar fissures in children have recently been reported and have been hypothesized to represent a novel pattern of bottom-of-fissure dysplasia. ${ }^{1}$ On the basis of our observation of a similar imaging pattern in patients -including some with initially normal scan findings-who displayed a depth of cerebellar fissure abnormality attributable to ischemic injury, we sought to review our institutional experience. We report a series of 23 patients with a similar distribution and appearance of cerebellar signal abnormality attributable to watershed injury.

\section{MATERIALS AND METHODS}

The study was approved by our institutional review board, and on the basis of minimal risk, the need for written informed consent

Received December 17, 2019; accepted after revision March 16, 2020. From the Departments of Radiology (J.N.W., D.W.W.S., G.I., F.P.) and Pediatrics (D.D.), Divisions of Developmental and Genetic Medicine, University of Washington and Seattle Children's Hospital, Seattle, Washington.

Please address correspondence to Jason N. Wright, MD, M/S MA.7.220, Seattle Children's Hospital, PO Box 5371, Seattle, WA, 98105; e-mail: jnixon@uw.edu

http://dx.doi.org/10.3174/ajnr.A6532 was waived. Twenty-three patients with MR imaging findings of focal T2 prolongation in the cerebellar gray matter at the depth of the fissures were identified by querying our local radiology data base for the past 15 years and are included in this review. Retrospective analysis of the MR imaging studies was performed by 2 subspecialty board-certified pediatric neuroradiologists with 8 (J.N.W.) and 7 (F.P.) years of experience. Imaging datasets were qualitatively analyzed for the characteristics and distribution of signal abnormality within posterior fossa structures, the presence and distribution of volume loss, the presence of abnormal contrast enhancement, and the presence and pattern of supratentorial injury. Patient demographics, clinical histories, and ultimate clinicoradiologic diagnoses were determined by chart review.

\section{RESULTS}

\section{Patients}

Twenty-three patients with characteristic abnormal cerebellar signal were included in our retrospective analysis, of which 16 were males and 7 females ( 1 proportion $Z$-test; $z=1.877 ; P=.06$ ). Age 

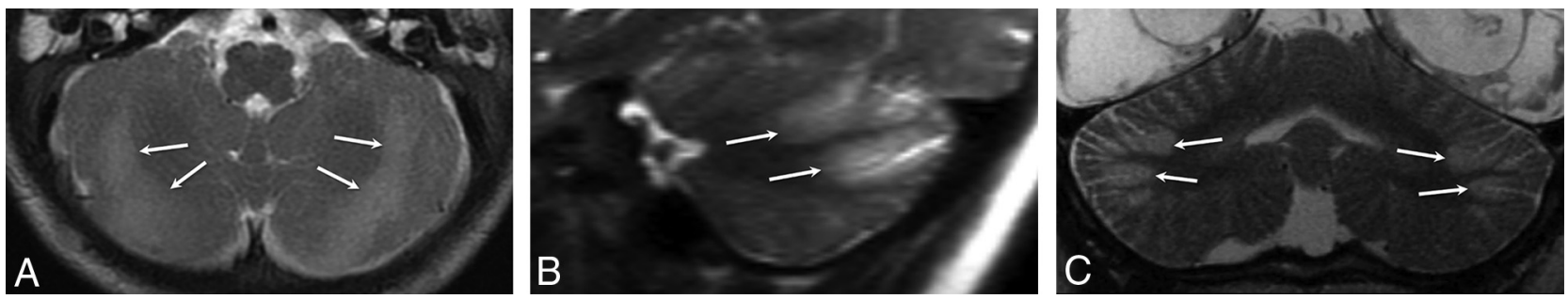

FIG 1. Axial T2WI from patient $2(A)$, parasagittal T2WI from patient $8(B)$, and a coronal T2WI from patient $6(C)$ demonstrate the characteristic appearance and location of cerebellar watershed injury. There is T2 prolongation greatest in the gray matter at the depths of the cerebellar fissures (white arrows) within the deep cerebellar watershed territory.

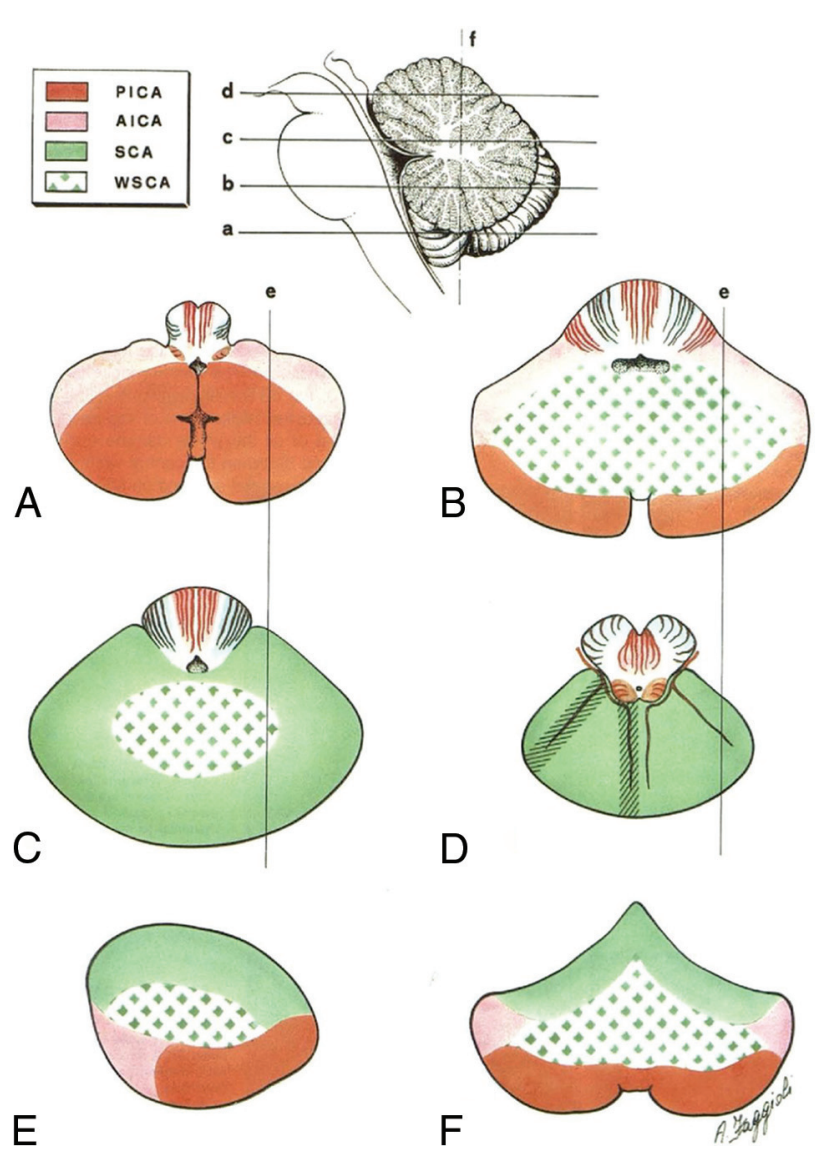

FIG 2. Schematic drawing of the vascular territories of the cerebellum. Note the central distribution of the cerebellar watershed areas (WSCA) at the junction of PICA, AICA, and SCA. The distribution of MR imaging signal abnormality in our case series corresponds to cerebellar watershed regions, best demonstrated by comparing the axial $(B)$, parasagittal $(E)$, and coronal $(F)$ schematic images with multiplanar MR images in Fig 1. Modified from Savoiardo et al. ${ }^{\text {? }}$

at the first MR imaging examination demonstrating cerebellar signal abnormality ranged from 2 months to 18 years, with a mean of 4.4 years and a median of 1.2 years. The mean age for patients with acute-phase imaging $(n=16)$ was 3.2 years; and for acute-phase imaging excluding posterior reversible encephalopathy syndrome (PRES) $(n=13)$, it was 0.7 years. Final clinicoradiologic determination of the etiology of brain injury was hypoxic-ischemic injury (HII) in 17 patients, PRES in 3 patients, and indeterminate in 3 patients. Among patients with HII, 5 cases were secondary to abusive head trauma; 4 , to cardiorespiratory arrest; 3 , to perinatal birth injury; 2 , to profound septic shock; 2 , to perioperative hypotension; and 1, to accidental asphyxiation. Ten patients had follow-up imaging at least 1 year after the initial MR imaging demonstrating cerebellar abnormality, with a median follow-up of 64 months (range, 15-142 months). Five patients had past MR imaging studies available, obtained before the first MR imaging that demonstrated the cerebellar abnormality.

\section{Infratentorial Imaging Findings}

The key imaging feature was T2 and FLAIR hyperintensity and T1 hypointensity confined to the gray matter and immediate subjacent white matter at the depths of the cerebellar fissures (Fig 1). The findings were best seen on coronal and parasagittal fluid-sensitive sequences. In all patients, bilateral hemispheric involvement was present, with injury typically involving multiple fissures centered at the expected location of the borderzone between the superior cerebellar artery (SCA), AICA, and PICA (Fig 2). The cerebellar tonsils were injured in 39\% (9/23) of cases. The cerebellar vermis was spared in $91 \%(21 / 23)$ of patients. Five of the 23 patients had prior imaging studies, all of which demonstrated a normal cerebellar cortex (Fig 3).

Imaging was available for $16 / 23$ patients during the acute phase of injury, defined as $<7$ days from the suspected clinical event. All patients demonstrated normal thickness or a mildly thickened cerebellar cortex (Figs 3 and 4). Eighty-one percent (13/16) of patients demonstrated acute diffusion restriction (12 with an eventual clinical diagnosis of HII, 1 of unknown etiology) (Fig 4A-C), while 19\% (3/16) of patients demonstrated no diffusion abnormality (all with an eventual clinical diagnosis of PRES) (Fig 4D-F). The 3 patients with PRES were imaged in the acute phase only, with no imaging follow-up available.

Imaging was available for $7 / 23$ patients in the subacute phase of injury, defined as 7-30 days from the suspected clinical event. Three of 7 patients (patients 4, 18, and 22) demonstrated intrinsic T1 signal hyperintensity at the depths of the cerebellar fissures (Fig $5 A$ ). In 1 case, this was demonstrated to have resolved on a follow-up study performed 1 month later (patient 22) (Fig 5B). One patient who underwent contrast-enhanced MR imaging in the subacute phase demonstrated focal contrast enhancement in the areas of cerebellar injury (patient 18).

Imaging was available for $15 / 23$ patients in the chronic phase of injury, defined as $>30$ days from the suspected clinical event. All had variable thicknesses of T2 prolongation without diffusion restriction in the cerebellar cortex and the immediate subcortical 

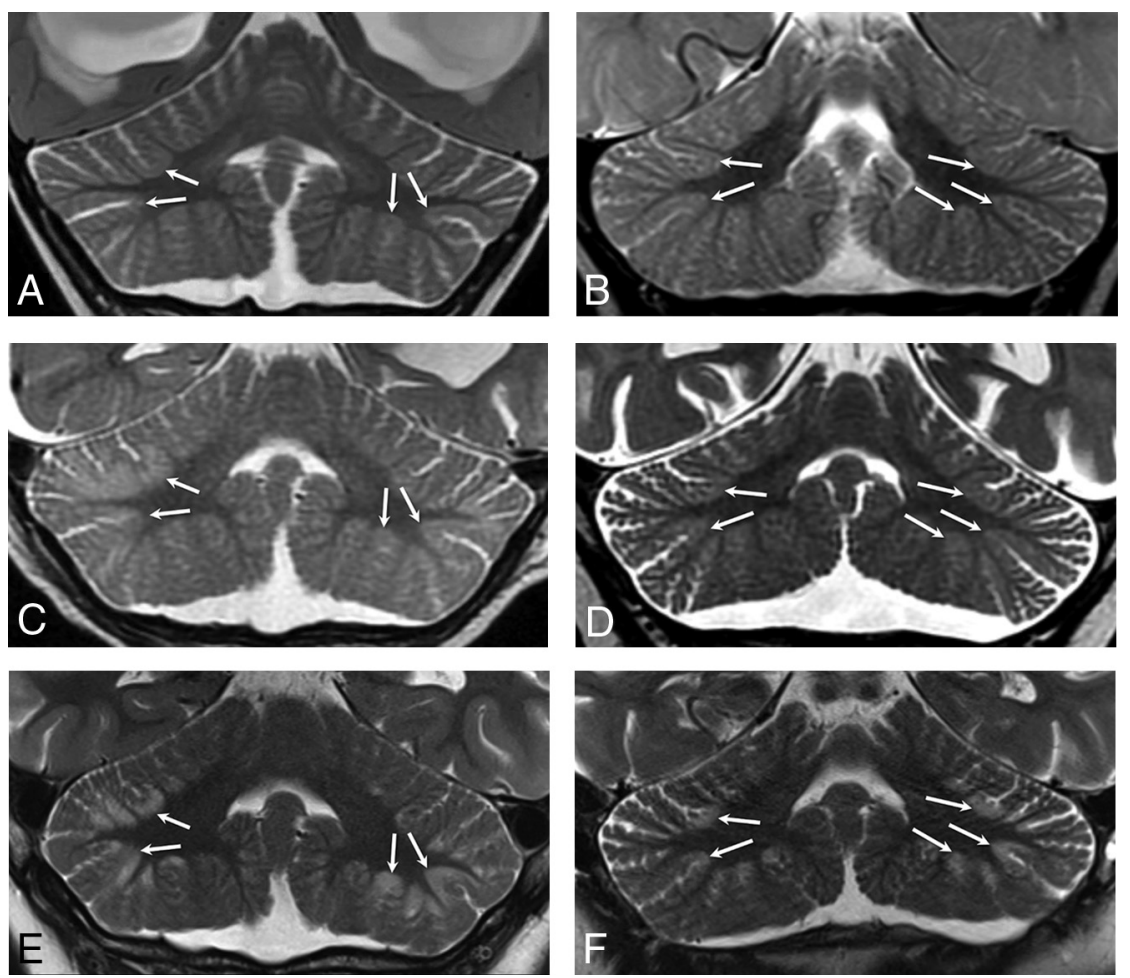

FIG 3. Coronal T2WI from patient $4(A, C$, and $E)$ and patient $11(B, D$, and $F)$ demonstrates the appearance of newly developed acute $(C$ and $D)$ and subsequent chronic $(E$ and $F$ ) phase injury in previously normal cerebella ( $A$ and $B$ ). White arrows highlight multipe sites demonstrating progressive development of typical watershed injury in previously normal gray matter.
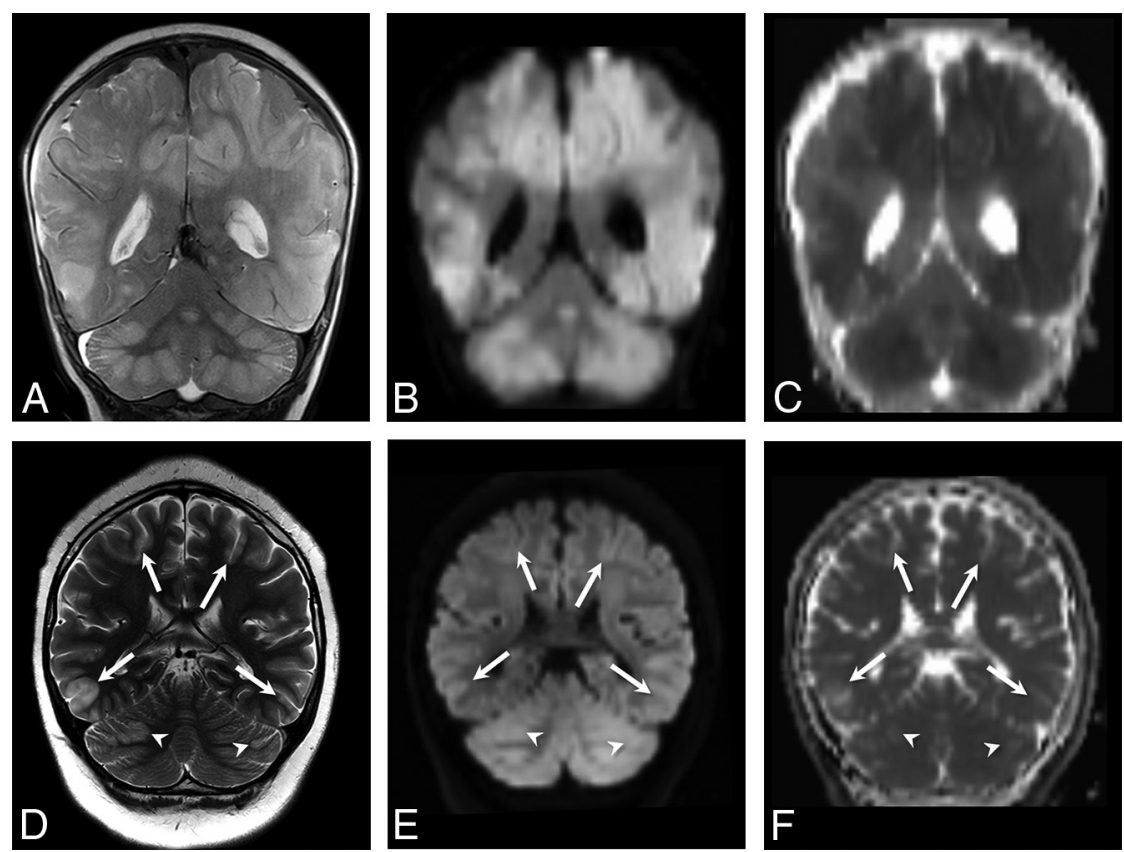

FIG 4. Coronal T2WI $(A)$, coronal DWI $(B)$, and coronal ADC map $(C)$ from patient 18 demonstrate acute cerebellar and supratentorial injury with $\mathrm{T} 2$ prolongation and restricted diffusion in the setting of HII. Coronal T2WI (D), coronal DWI (E), and coronal ADC map $(F)$ from patient 9 demonstrate acute cerebellar (white arrowheads) and supratentorial (white arrows) injury without diffusion restriction in the setting of PRES. white matter (Fig 6). Seven of 15 patients had marked cortical volume loss with minimal residual T2 hyperintensity (Fig 6A, $-B$ ), while the remaining 8 demonstrated a relatively thick layer of T2 hypersignal involving the foliar cortex without substantial volume loss (Fig 6C, -D). Subtle fissural prominence and mild foliar volume loss were identified in 14/15 patients. Ten patients had followup for $>1$ year after the initial MR imaging demonstrating cerebellar signal abnormality, with a median followup interval of 5.3 years (range, 1.311.8 years). Cerebellar signal abnormality persisted in all cases and for up to 11.8 years in the case with the longest follow-up (patient 1). There were no discernable group differences in terms of the presence of supratentorial injury, overall injury extent or severity, the presence of acute-phase diffusion restriction, underlying etiology, or mean length of follow-up between patients with thick or thin T2 foliar hyperintensity in the chronic phase.

\section{Supratentorial Imaging Findings}

In the supratentorial brain, $87 \%(20 / 23)$ of patients demonstrated a classic parasagittal watershed pattern of cortical injury (Fig 7), with 11 with additional evidence of associated basal ganglia injury. Seventeen patients had watershed infarctions and a concordant clinical history of HII. The remaining 3 patients demonstrated a watershed pattern of injury typical of PRES. In all cases, the apparent timing of the supratentorial injury was consistent with the estimated timing relative to the suspected clinical event.

Three of 23 patients demonstrated no evidence of supratentorial watershed cortical injury. One (patient 11) demonstrated diffuse progressive cortical atrophy and a deep pattern of watershed injury in the supratentorial white matter, in the context of underlying drug-resistant chronic epileptic encephalopathy. The remaining 2 patients had no demonstrable supratentorial cortical injury. The first of these had a history of remote aortic stenosis and coarctation repair, and the second had end-stage renal disease 
complicated by chronic hypotension and respiratory failure, both of which may have predisposed to recurrent hypoperfusion events that were clinically unsuspected. Of the 3 patients without supratentorial cortical watershed injury, 2 had prior imaging demonstrating normal cerebella (patients 10 and 11).

\section{DISCUSSION}

We hypothesize that the pathologic basis for the stereotyped depthof-cerebellar-fissure MR imaging findings in our case series is cerebellar watershed injury for the following reasons: 1) location at the deep borderzone between the cerebellar artery vascular territories, 2) association with a classic watershed pattern of supratentorial cortical injury, 3) frequent acute-phase diffusion restriction with expected evolution across time, and 4) frequent late foliar volume loss with fissural prominence. Furthermore, among patients with MR imaging performed before the suspected clinical insult, the cerebellar abnormalities were not previously present, indicating an acquired insult rather than a congenital malformation. Diaschisis
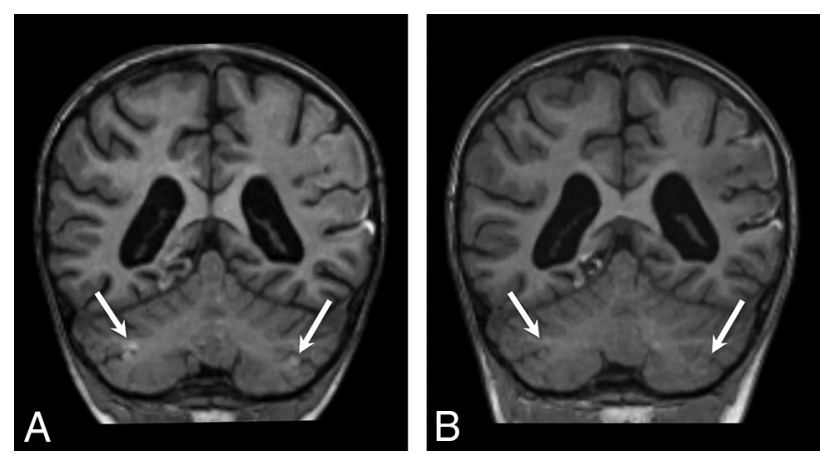

FIG 5. Coronal TTWI from patient 22 in the subacute $(A)$ and chronic (B) phases demonstrates focal $T 1$ hyperintensity in the bilateral cerebellar watershed (white arrows in A), consistent with laminar necrosis and resolved within 1 month on follow-up (white arrows in $B$ ).
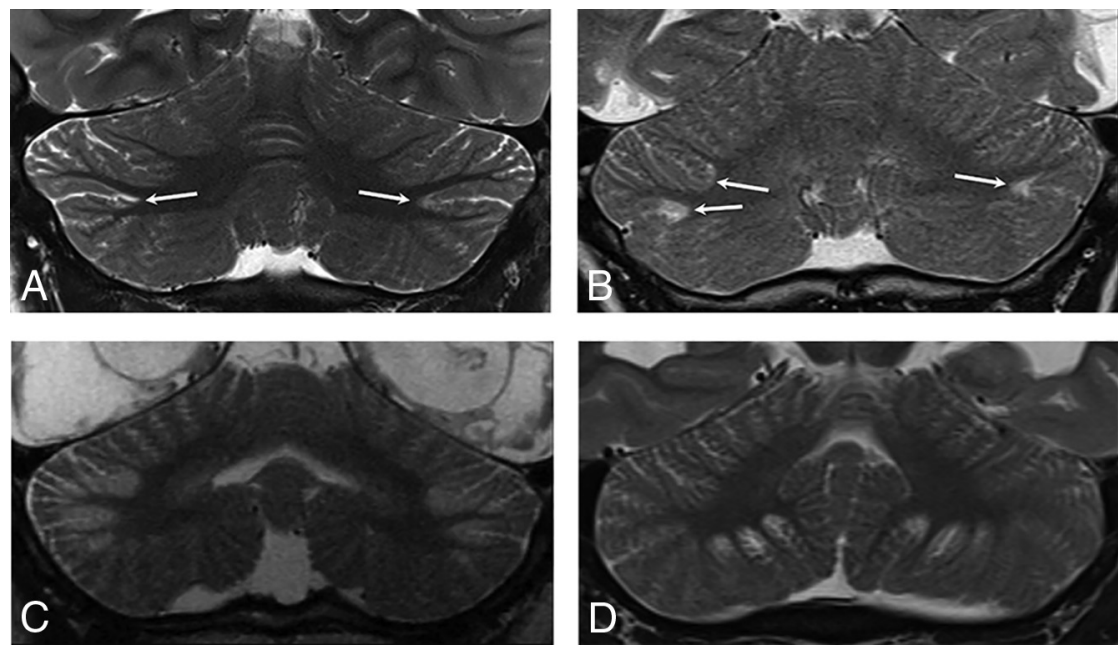

FIG 6. Coronal T2WI from patients during the chronic phase. Patients $1(A)$ and $2(B)$ demonstrate T2 prolongation with relatively marked gray matter volume loss at the sites of prior injury (white arrows). Patients $7(C)$ and $10(D)$ demonstrate a thick lamina of gray matter T2 prolongation centered at the depths of the cerebellar fissures in an arterial watershed distribution. See also Figs $1 C, 3 E$, and $3 F$ for additional examples. secondary to the supratentorial injury was considered an unlikely contributor to the observed infratentorial injury pattern, due to the simultaneous evolution of brain injury in both locations and the multifocal rather than diffuse nature of the cerebellar findings.

Two distinct etiologies of watershed injury were observed in our case series, including PRES and watershed infarctions in the setting of HII. The cortical injury in PRES is poorly characterized at the cellular level but is presumed secondary to abnormal perfusion in the setting of disturbed autoregulation. As is typical of this entity, acute-phase imaging demonstrated T2 prolongation associated with facilitated diffusion. PRES has a strong and welldescribed predilection for vascular borderzones in the supratentorial brain. While cerebellar involvement has been reported, its distribution has not been well-characterized. ${ }^{2}$

A classic parasagittal distribution of supratentorial watershed infarctions is well-recognized, with preferential involvement of the end-arterial borderzones between the anterior, middle, and posterior cerebral artery vascular territories. Cerebellar watershed infarcts remain less well-described, with scattered case reports and case series describing predominantly embolic watershed infarcts in adults. ${ }^{3-6} \mathrm{~A}$ few authors have emphasized the distinction between superficial cerebellar borderzone embolic infarcts and deep cerebellar watershed infarcts, the latter related to hypoperfusion and centered at the interface between the penetrating arteries of the cerebellar arteries. ${ }^{7-10}$

Although some authors have asserted that deep watershed infarcts predominantly affect the deep cerebellar white matter, several lines of evidence suggest that focal injury to the gray matter at the depth of the cerebellar fissures is, in fact, the expected pattern. In a primate model of induced cerebral hypoperfusion, ${ }^{11,12}$ cerebellar injury in the watershed between the SCA and PICA was noted in 7/15 adult rhesus monkeys, in conjunction with classic supratentorial watershed injury. The injury predominantly affected cortical gray matter at the depths of the cerebellar fissures. Depth of fissure gray matter injury was also noted on gross and histopathologic evaluations of human brains after hypoxic injury, ${ }^{13}$ in a pattern exactly corresponding to the distribution of injury in our case series. This distribution may reflect the unique angioarchitecture of the penetrating arteries in the cerebellum, analogous to the propensity for depth-ofsulcus cortical injury in neonates with HII reported by Takashima et al. ${ }^{14}$

Sparing of the cerebellar vermis was a notable feature in our patients, possibly because the arterial supply to the vermis is almost entirely derived from the SCA, with relatively minor contributions from the inferior cerebellar arteries. ${ }^{15}$ In contrast, cerebellar tonsillar injury was observed in more than one-third of patients in our series, likely reflecting its location at the borderzone between the AICA and PICA territories. ${ }^{15}$ 

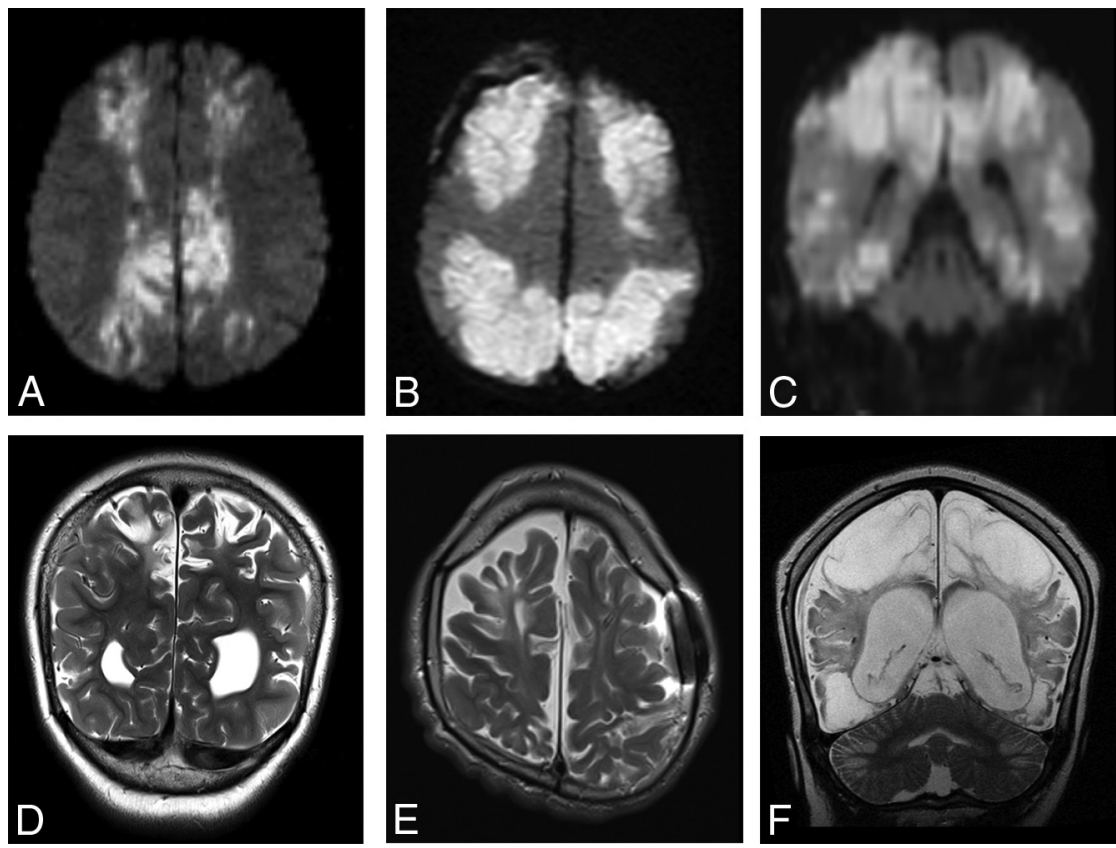

FIG 7. Findings of both acute and remote supratentorial watershed injury in patients $1-6(A-F$, respectively). Acute watershed infarcts with signal abnormality on axial ( $A$ and $B$ ) and coronal $(C)$ DWI in a characteristic parasagittal distribution. Subacute or remote watershed infarcts with hyperintensity and parenchymal volume loss on coronal ( $D$ and $F$ ) and axial $(E)$ T2. present in some or all of their cases, or that cerebellar watershed injury and bottom-of-fissure dysplasia share a common imaging appearance. Although they considered the possibility of cerebellar watershed injury as the etiology of the imaging findings in Poretti et $\mathrm{al}^{1}{ }^{1}$ they ultimately concluded that lack of substantial white matter involvement, variability of distribution of involved fissures, and stability with time all argued against this possibility. It is clear from our patient seriescorroborated by the available experimental neurophysiology and neuropathology literature - that the foliar gray matter at the depths of the fissures is an expected location of cerebellar watershed injury, with minimal or absent white matter involvement. ${ }^{11-13}$ Variable fissural involvement is expected because it reflects the relatively variable vascular territories and shifting borderzones of the cerebellar arteries. ${ }^{15}$ Furthermore, stability with time in the chronic phase, often with unexpectedly subtle vol-

Our patient series comprised a disproportionate number of male patients, though this finding did not reach statistical significance regarding variance from an expected population proportion of $50 \%$ male. This may, in part, be related to the number of cases of HII secondary to abusive head trauma in our series, which is known to disproportionately affect male patients. ${ }^{16}$ Haas-Lude et $\mathrm{al}^{17}$ recently reported a strikingly similar pattern of cerebellar injury in 2 patients with abusive head trauma, both with a supratentorial watershed pattern of HII. It would be interesting to investigate the prevalence of this finding in a larger cohort of patients with suspected abusive head trauma to better understand its predictive value above and beyond the presence of HII more generally.

Our case series is among the first to describe the characteristic appearance of cerebellar watershed injury in children. Recognition of this pattern of injury is important for identifying patients with HII or PRES and for avoiding misdiagnosis. For example, 1 of our patients was originally misdiagnosed with cerebellitis based on abnormal cerebellar T2 hyperintensity (patient 10), delaying definitive diagnosis. Similarly, Mills et $\mathrm{al}^{18}$ reported a case of "severe cerebellitis" in a child with supratentorial watershed infarcts following methadone poisoning. The imaging findings in that case are strikingly similar to those in our case series, raising the possibility of watershed cerebellar injury rather than cerebellitis.

A second patient in our case series was initially diagnosed with bottom-of-fissure dysplasia; however, a prior MR imaging subsequently became available demonstrating a normal cerebellum, indicating an acquired rather-than-congenital lesion. The similarity between our reported imaging findings and those of Poretti et $\mathrm{al}^{1}$ suggests that unrecognized watershed injury may have been ume loss, was the norm in our series.

There are several limitations to our analysis, primarily reflecting the small sample size and retrospective design. We were unable to assess the clinical implications of cerebellar watershed injury in our case series because of the small and heterogeneous sample. Our study does not permit an analysis of the prevalence of cerebellar imaging findings among children with supratentorial watershed injury. It is also possible that a subset of our cases may represent a dysplastic process as suggested by Poretti et $\mathrm{al}{ }^{1}$ particularly those without corroborative supratentorial findings. However, 5 of our patients had a prior MR imaging study demonstrating a normal cerebellum, including 2 of the 3 patients without clear supratentorial cortical watershed injury, arguing against the likelihood of a developmental abnormality.

\section{CONCLUSIONS}

We have demonstrated a stereotyped pattern of injury that is most plausibly attributable to cerebellar watershed injury. This report extends the reported imaging findings in watershed cortical injury related to HII and PRES, which are an important diagnostic consideration when cerebellar dysplasia and inflammatory pathologies such as cerebellitis are being entertained.

\section{REFERENCES}

1. Poretti A, Capone A, Hackenberg A, et al. Cerebellar bottom-of-fissure dysplasia: a novel cerebellar gray matter neuroimaging pattern. Cerebellum 2016;15:705-09 CrossRef Medline

2. Bartynski WS, Boardman JF. Distinct imaging patterns and lesion distribution in posterior reversible encephalopathy syndrome. AJNR Am J Neuroradiol 2007;28:1320-27 CrossRef Medline 
3. Amarenco P, Kase CS, Rosengart A, et al. Very small (border zone) cerebellar infarcts: distribution, causes, mechanisms and clinical features. Brain 1993;116:161-86 CrossRef Medline

4. Yin WM, Nagata K, Satoh Y, et al. Infratentorial infarction: correlation of MR findings with neurological and angiographical features. Neurol Res1994;16:154-58 CrossRef Medline

5. Benjumea V, Vélez A, Hernández $\mathrm{O}$, et al. Infartos limítrofes cerebrales y cerebelosos asociados a compromiso hemodinámico: reporte de caso y revisión de la literature. Acta Neurol Colomb 2014;30:342-45. http://www.scielo.org.co/pdf/anco/v30n4/v30n4a16.pdf. Accessed April 6, 2020

6. Zheng M, Sun AP, Sun QL, et al. Clinical and imaging analysis of a cerebellar watershed infarction. Chinese Medicine 2015;06:54-60 CrossRef

7. Savoiardo M, Bracchi M, Passerini A, et al. The vascular territories in the cerebellum and brainstem: CT and MR study. AJNR Am J Neuroradiol 1987;8:199-209 Medline

8. Cormier PJ, Long ER, Russell EJ. MR imaging of posterior fossa infarctions: vascular territories and clinical correlates. Radiographics 1992;12:1079-96 CrossRef Medline

9. Amarenco P. Cerebellar infarctions and their mechanisms. Rev Neurol (Paris) 1993;149:728-48 Medline

10. Caplan LR. Cerebellar infarcts: key features. Rev Neurol Dis 2005;2:51-60 Medline
11. Brierley JB, Excell BJ. The effects of profound systemic hypotension upon the brain of M. rhesus. Brain 1966;89:269-98 CrossRef Medline

12. Brierley JB, Brown AW, Excell J, et al. Brain damage in the rhesus monkey resulting from profound arterial hypotension, I: its nature, distribution and general physiological correlates. Brain Res 1969;13:68-100 CrossRef Medline

13. Graham DI. Pathology of hypoxic brain damage in man. J Clin Path Suppl (R Coll Pathol) 1977;11:170-80 CrossRef Medline

14. Takashima S, Armstrong DL, Becker LE. Subcortical leukomalacia: relationship to development of the cerebral sulcus and its vascular supply. Arch Neurol 1978;35:470-72 CrossRef Medline

15. Icardo JM, Ojeda JL, Garcia-Porrero JA, et al. The cerebellar arteries: cortical patterns and vascularization of the cerebellar nuclei. Acta Anat (Basel) 1982;113:108-16 CrossRef Medline

16. Selassie AW, Borg K, Busch C, et al. Abusive head trauma in young children: a population-based study. Pediatr Emerg Care 2013;29:28391 CrossRef Medline

17. Haas-Lude K, Roulet-Perez E, Döbler-Neumann M, et al. Cerebellar lesions in pediatric abusive head trauma. Eur J Paediatr Neurol 2019;23:604-08 CrossRef Medline

18. Mills F, MacLennan SC, Devile CJ, et al. Severe cerebellitis following methadone poisoning. Pediatr Radiol 2008;38:227-29 CrossRef Medline 